\title{
Fast Tracking Algorithm with Borders 1-D Histogram Correlation
}

\author{
María Curetti, Member, IEEE, Santiago Garcia Bravo, Member, IEEE, \\ Gabriela Soledad Arri, and Ladislao Mathé, Member, IEEE
}

\begin{abstract}
This paper presents a fast algorithm for object tracking in an image sequence. It is a method that models the borders of the image as onedimensional histograms which are then used instead of templates in the matching procedure. The algorithm models the item being tracked as well as the background in the vicinity so as to then suppress it. It uses cross correlation to find the best match and weighted average to renew the model.
\end{abstract}

Keywords: Image matching, Image sequence analysis, Video signal processing.

\section{Introduction}

Object tracking through image sequences is one of the problems of image processing most often studied. Besides its inherent worth, tracking is the previous step for any navigation system based on images. To this end, a wide variety of algorithms that model the object of interest and then look for it in the image are used. One of the first methods to be applied in object tracking is template matching. This algorithm takes a known intensity mask of the item being tracked and compares it with the image until it finds the place where their correlation takes its maximum value. [1], [2], [3], [5].

This method presents a series of problems, among them, the changes that the object appearance suffers with time is one of the most significant ones. Variations in the light and in the shape of the image must be taken into account among other case scenarios. To overcome this, a variety of approaches with different amount of computational load have been employed. [4], [7].

Updating the template after a certain amount of frames is a basic method often applied to outline a valid solution for some of those problems. The main failure of this algorithm is that the object may drift from the mask. To keep the element centered in the template, in [6] the approach proposed is to preserve the first mask and use it in the new template to prevent the drift. Transitory occlusion may occur deteriorating the mask in algorithms that lack robustness. In [8], Kalman filters are used to estimate the intensity of the new template to improve robustness against occlusions. In newer approaches, in [10] for example, the step of matching based on local brightness was replaced with a search for a model. The main reason that leads to this new tactic is the limitation in the brightness mask's constancy originated by light appearance changes.

The objective of this work is to model the object through a set of one-dimensional histograms that describe the borders of the tracked object; then these profiles are used to find the same border distribution in the next frame. Here "border" is defined as the 
dividing line or frontier between regions with different intensity on the image. This one-dimensional model is not fixed and allows amendments to take into account the changes in the appearance and size of the object. To avoid the disturbance caused by the background borders, their profiles in the vicinity of the target are modeled and suppressed. These models are not only continuously updated but they also drift from the target position; therefore, they must be correlated before suppression. With heterogeneous backgrounds, this last step is not only essential but also enables the algorithm to estimate the relative movements of the object, taking the background as reference. The main advantage of this algorithm is that, as it models the mask (section of the picture that contains the target) through 1-D signals, the computational load grows proportionally to the object's size to the first power instead of the square power, as it usually happens. Furthermore, the background suppression avoids erroneous changes of the mask's size that can arise when borders are the basis of this choice.

In [9], the tracked object pixel's color distribution is also modeled by a histogram. Then the central computational module, based on mean shift iterations, finds the most probable target position in the current frame. The histograms employed in that work, unlike the ones used in this paper, had bins with no relation to the position of the pixel classified, thus making the description of the target appearance generic. With a $240 \times 360$ image and a variable mask from $25 \times 39$ to $9 \times 9$ following the changes of the object, a processing speed of more than $660 \mathrm{fps}$ is achieved in an Intel(R) Core(TM) i7-2600 CPU $3.4 \mathrm{GHz}$.

\section{System Outline}

The tracking system proposed has an initialization stage and five sequential stages that iterate for every frame. Before the sequence of images is processed, the first location and the target size must be input, and the models of the background and target are initialized. The first step in each iteration is to model the region near the location expected for the object in the new frame. Then, the drift of the background is estimated correlating its model with the borders extracted from the frame. Next, the background model is subtracted from the total image model calculated in the new sample. After that, the location that maximizes the correlation with the model of the target is found. Finally, both, the background and target models are updated with a weighted average, and everything is ready for the next iteration.

At the end of each iteration, the algorithm determines whether the mask needs to grow or shrink, and if the target is centered. The algorithm calculates a set of coefficients that reflect the presence of borders near the boundaries of the mask. Then, it compares them to the threshold values that change depending on the target size. Thus, the object will not drift from the mask and the whole target will be contained within.

Prior to explaining each stage of the system, it is necessary to define the model proposed to describe the borders in the image. 


\section{$3 \quad$ Model}

This model's main objective is to locate the borders of the image through a set of onedimensional histograms that accumulate the number of pixels corresponding to the target border in each column and row. The system selects four kinds of borders, namely vertical and horizontal borders, which may be positive or negative. This leaves us with eight histograms, four that integrate all the data in a column in one value and four that summarize the rows information.

The first step to calculate this model is to locate the borders in the image. To confirm whether a pixel belongs to any of the target borders proposed, the difference between its intensity and its neighbor's intensity is calculated. Then, the absolute value is compared to a threshold. If the value is greater than this threshold, the pixel will be classified depending on the sign of the difference and the location of the neighbor. This method is applied to the pixels located to the right and below the evaluated pixel Consequently, a pixel will be classified as a positive border if its intensity is higher than the intensity of the one in the row or column immediately next to it, as shown in this expressions:

$$
\begin{gathered}
I_{r, c}-I_{r+1, c}>\alpha \Rightarrow \text { hor.border }(+) ; I_{r, c}-I_{r+1, c}<\alpha \Rightarrow \text { hor.border }(-) \\
I_{r, c}-I_{r, c+1}>\alpha \Rightarrow \text { vert. border }(+) ; I_{r, c}-I_{r, c+1}<\alpha \Rightarrow \text { vert.border }(-)
\end{gathered}
$$

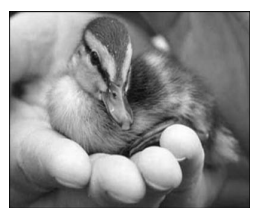

Fig. 1. Sample image

With this classification method, four matrices will be obtained with unitary value in the border locations. In Fig. 2 a representation of them can be observed.

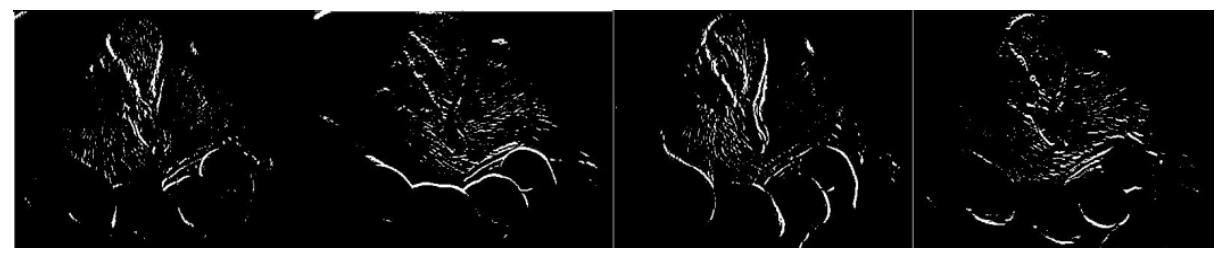

Fig. 2. Four classification matrices: the vertical borders on the first and third image, and a negative value on the first two images may be observed

From these matrices the computation of the histograms (Fig. 3) is simple.

If the matrices are called $M_{i}$, with $\mathrm{i} \in\{1,2,3,4\}$, then the calculation needed to complete the model would be:

$$
H_{c i}=\sum_{c} M_{i} ; H_{r i}=\sum_{r} M_{i}
$$



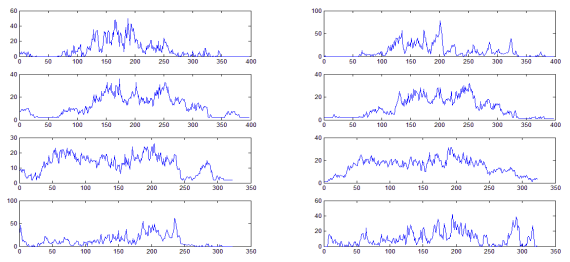

Fig. 3. Eight histograms obtained

\section{Initialization}

In this stage, it is important to define the target location and size in the first frame. Once these data are input, the system will estimate the target model (tgt) and the background model (bkg). It is convenient not to start with a mask larger than the target since it may incorporate more background borders than necessary. Then, the system will suppress the background borders from the object model, assuming that the target only covers a small section of the background and that the borders will continue inside with some homogeneity. A more detailed explanation of this stage procedure is included below.

First, the system will extract the section of the image centered on the target location. This section has to be twice as large as the mask. Next, all the pixels in this section are classified. Then, the model for the central region is calculated. This will be the first estimate of the target model. All the pixels in this central region are set to zero and then the remaining section is modeled as an approximation of "bkg".

To estimate the central values that belonged to the background and that have been erased, the horizontal borders interrupted by the target will be completed horizontally. The same approximation is applied to the vertical borders to finish "bkg".

Once the background model is estimated, the target model is modified, subtracting the pixels that were decided to belong to borders of the background from the histograms.

\section{$5 \quad$ Stages 1 to 3}

The first three stages are similar to the initialization. The main difference is that then the position was known.

The double sized section will be extracted from the vicinity of the estimated position, calculated from the previous positions. All the pixels from this extracted section are classified and modeled (tot). This model (b\&t) will include both background and target.

To subtract the modeled background (bkg) from this model (b\&t), first the background drift must be determined. Instead of working with the total model the central region in the matrices is turned to zero, and the same estimations made in the initialization are applied to estimate an approximate model of the background alone (bap) in the new frame. The region that must be turned to zero is bigger than the 
target mask size because the current location is just an estimation that has uncertainty.

To establish the relative position of the two signals that makes the match maximum, the cross correlation method is adopted. This technique calculates for each relative position the scalar product of the displaced signals.

$$
C_{u}=\sum_{x} f_{x} t_{x-u}
$$

Before the cross correlation is applied, the mean of each vector should be subtracted. Otherwise, the relative position that maximizes $C_{u}$, may not be the best match. For instance, if a histogram, with peaks and valleys, is correlated with a signal composed of this same histogram next to other vector of the same size, but with all its elements equal to the maximum of the histogram; the relative position that will maximize the result will be the one that aligns the first signal to the second part of this assembled signal. This can be avoided with the mean subtraction.

$$
\begin{gathered}
t_{x}^{\prime}=t_{x}-\bar{t} \\
f^{\prime}{ }_{x}=f_{x}-\bar{f} \\
\gamma_{u}^{\prime}=\sum_{x} f^{\prime}{ }_{x} t^{\prime}{ }_{x-u}
\end{gathered}
$$

Once the background drift that maximizes the summed cross correlations of each of the eight signals of the models (bkg and bap) is determined, it is used to subtract the background model (bkg) from the total model calculated at the beginning (b\&t). As a result, an estimated model (tonly), where only the target borders are modeled, is obtained for the current sample.

\section{$6 \quad$ Stage 4 and 5}

In the fourth stage, the correlations of the eight signals of the models "tgt" and "tap" are calculated and added. The resulting value is used to determine a more accurate position of the target in the current frame.

The last stage, where the models (bkg and tgt) are updated, is a very important one. To perform the update, weighted averages are used, and the drift is taken into account. To update "tgt" the model used is "tap", because it's the estimated model of the target alone. A new model (bkn) will be calculated to renew "bkg", this can be obtained from the difference between the overall model (b\&t) and the target (tgt), in the position estimated during the last stage. The coefficients that establish the update rate are to be picked carefully. The weight given to the refreshing models should be low enough so as not to add erroneous information, but the renewal must be quick enough so as to follow the changes in the object and improve the model.

$$
\begin{aligned}
\text { fond }_{m} & =\text { fond }_{m-1}^{\prime} \times(1-\beta)+\text { totr }_{m}^{\prime} \times \beta \text { with } \beta<1 \\
\text { yyu }_{m} & =y y u_{m-1}^{\prime} \times(1-\varepsilon)+\operatorname{totp}_{m}^{\prime} \times \varepsilon \text { with } \varepsilon<1
\end{aligned}
$$

Before the weighted average is calculated, the models must be centered and sectioned considering the misalignment and changes in size. In the background model (bkg), 
new values that were not modeled in the previous step may be modeled; these are taken from the refreshing model directly (without the previous weighting).

\section{$7 \quad$ Resizing and Centering}

This stage allows the model to follow the changes in the size of the object, and it keeps the object centered. Keeping the model as small as possible is useful to avoid entering erroneous information and to decrease the computational load. But, if the model does not include the whole object, some information will not be taken into account and the chances of finding the target are lower.

To decide if the model must grow, every histogram will have its extreme values close to zero (there are no borders in the limit of the mask). If the sum of these extreme values exceeds a certain threshold the model will grow, adding two zeros to the extremes of the four histograms that follow the expanded dimension. It is worth mentioning that the change in size of each dimension is independent.

To resolve shrinking procedure, once we verified that the model will not grow, the values to be checked are those that are next to the extreme ones. If the sum of all these values does not exceed another threshold, that must be lower than the last one, the model will shrink. The extreme values of each of the four histogram of the dimension that is decreasing will be removed. If the threshold is not smaller than the one to which the extreme values were compared, then in the next frame this model will probably grow and this oscillation may continue for a while.

To ensure that the model will follow the size of the object; there is one more situation that must be considered. If the profiles are not centered, the target may shrink and the condition verified to diminish the model can be false. To prevent that from happening, the first three and the last three values of the model are added separately. If one of these trios is below the threshold picked in the last case, the model will shrink by removing the two values in that extreme.

Lastly, it should be pointed out that the threshold should depend on the dimensions of the mask. In this system, a percentage of the size of the dimension that is not being evaluated is used as threshold. It is logical to pick this value because it is also a percentage of the maximum value the histogram can have for this dimension.

\section{$8 \quad$ Experimental Results}

This tracking system was designed to find the position of a flying plane; it works as a module of a camera control project. The position of the tracked object in the image is the input of a control system in charge of centering the aircraft in the frame. Thus, the camera will be able to use the optic zoom to its full extent, preventing the target from escaping from the frame. It can only work correctly when applied in objects that fill a small region of the image (60x60 pixels top) that move relative to the background.

To test this algorithm effectiveness, its behavior was studied in two sequences of images: one of a UAV (Unmanned Air Vehicle) taking off and one of a flying plane. These sequences were taken from videos captured previously during another stage of 
this project's development. To evaluate the obtained results, they were contrasted with the ones attained by using two template matching algorithms: one that simply uses a fixed template and the other with adapting template. During the experience, the threshold values where adjusted so as to not lead to unstable behavior (such as size oscillation, continuous growth or shrinking).

The first sequence taken from the video contains 1500 frames, during the sequence, the UAV shape and its size change drastically. The adapting template technique and the proposed algorithm, both succeed in finding the UAV during the whole succession (Fig.4). The one with the fixed template fails due to the object sudden changes (Fig. 5 (a)). The rate of the adapting template method was of $256 \mathrm{fps}$, while the profile based algorithm processed 660 images per second. During the second sequence the results where similar, except for the algorithm with the fixed template that this time could follow the target.

Besides the position of the plane for each frame, the system described in this paper has other results that are worth mentioning. In Fig. 5 (b), the horizontal background's accumulated drift is plotted. The plane acceleration is perceptible to the eye. In Fig. 6, the evolution of one of the horizontal signal of background model is shown; here the relative movement is also clear. Finally, the evolution of one of the target model histogram is presented in Fig. 7. Here the model's shrinking accompanying the target changes in size is visible. As the model's histogram always starts in the first line, when the number of bins diminishes, small stepped lines appear in the otherwise gradual changes of the model.
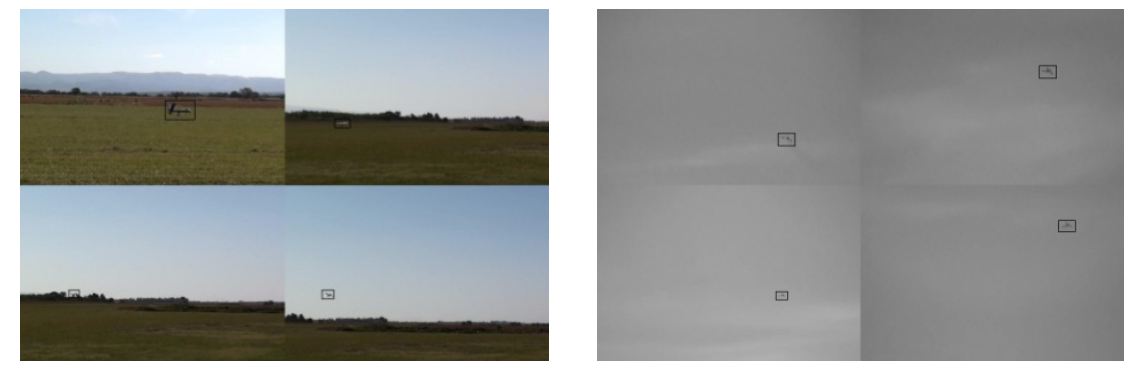

Fig. 4. Samples trough the sequence first and second sequence

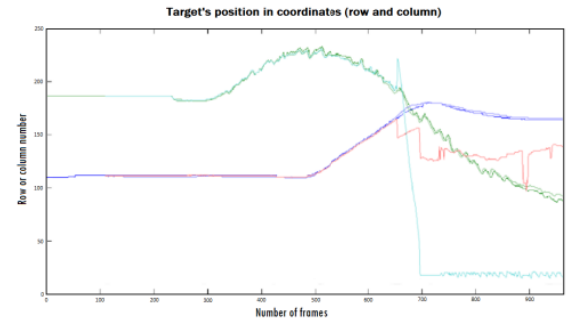

(a)

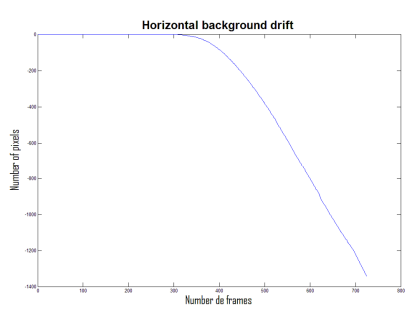

(b)

Fig. 5. (a) Position versus frames, (b) accumulated horizontal drift ve_sus frames 


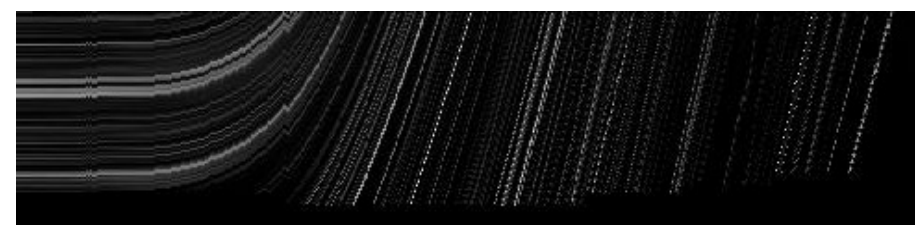

Fig. 6. Background model evolution

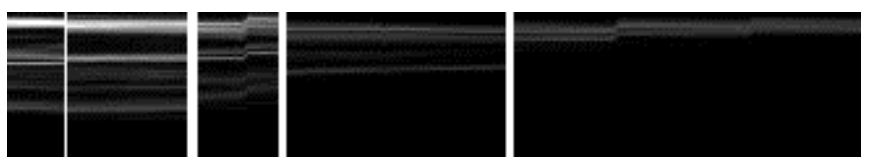

Fig. 7. Target's model evolution

\section{Conclusions}

The method proposed in this paper to track an object through a sequence of images, uses one-dimensional signals to model the borders in the image and improve the processing speed. It applies procedures of gradual renewal for the models, size adjustment and centering of the object. It also models the background, to suppress it in the new frame, and it makes trajectory predictions. It can run in real-time with images of 360x240 pixels of resolution and at frequencies of up to $660 \mathrm{fps}$ (2.5 times faster than the results obtained with the adaptive mask method). It is robust to changes of the target's appearance, and partial occlusions.

In the future, the performance of this algorithm will be evaluated in sequences where the background is not static. For example, a scenery with trees being moved by the wind.

Acknowledgment. This work was supported by the Ministry of Defense of the Nation, within the framework of the project PIDDEF 019/2010, in charge of Ing. L. Mathé.

It was developed in the facilities of the "Centro de Investigaciones Aplicadas" dependent on the "Instituto Universitario Aeronáutico", Córdoba, Argentina.

\section{References}

1. Black, M., Jepson, A.: Eigen-Tracking: Robust Matching and tracking of Articulated Objects Using a View-Based Representation. Int'l. J. Computer Vision 36(2), 63-84 (1998)

2. Hager, G., Belhumeur, P.: Efficient Region Tracking with Parametric Models of Geometry and Illumination. IEEE Trans. Pattern Analysis and Machine Intelligence 20(10), 10251039 (1998)

3. Cootes, T., Edwards, G., Taylor, C.: Active Appearance Models. IEEE Trans. Pattern Analysis and Machine Intelligence 23(6), 681-685 (2001) 
4. Xiao, J., Kanade, T., Cohn, J.: Robust Full-Motion Recovery of Head by Dynamic Templates and Re-Registration Techniques. In: Proc. IEEE Int'l Conf. Automatic Face and Gesture Recognition, pp. 163-169 (2002)

5. Baker, S., Matthews, I.: Lucas-Kanade 20 Years on: A Unifying Framework. Int'l. J. Computer Vision 53(3), 221-255 (2004)

6. Matthews, I., Ishikawa, T., Baker, S.: The Template Update Problem. IEEE Trans. on Pattern Analysis and Machine Intelligence 26(6) (2004)

7. Zhong, Y., Jain, A.K., Dubuisson-Jolly, M.-P.: Object Tracking Using Deformable Templates. IEEE Trans. on Pattern Analysis and Machine Intelligence 22(5) (May 2000)

8. Nguyen, H.T., Worring, M., van den Boomgaard, R.: Occlusion robust adaptive template tracking. In: Eighth IEEE International Conference on Computer Vision (2001)

9. Comaniciu, D., Ramesh, V., Meer, P.: Real-Time Tracking of Non-Rigid Objects using Mean Shift. IEEE (2000)

10. Wu, Y., Fan, J.: Contextual Flow. In: IEEE Conference on Computer Vision and Pattern Recognition (2009) 